\title{
3 Research Square

\section{Triglyceride Glucose Index is Independently Associated with Aorta Intima-Media Thickness in Patients without Clinical Manifestations of Cardiovascular Disease}

\section{Süleyman ÖZBiçER ( $\square$ suleymanozbicer@gmail.com )}

University of Health Sciences, Adana Training and Research Hospital, Department of Cardiology, Adana, Turkey.

\section{Gülhan YÜKSEL}

University of Health Sciences, Adana Training and Research Hospital, Department of Cardiology, Adana, Turkey.

\section{Örsan Deniz URGUN}

University of Health Sciences, Adana Training and Research Hospital, Department of Cardiology, Adana, Turkey.

\section{Research Article}

Keywords: Arterial intima, Atherosclerotic plaques, Insulin resistance, Transesophageal echocardiography, Triglycerides

Posted Date: June 6th, 2022

DOl: https://doi.org/10.21203/rs.3.rs-1246442/v2

License: (1) (1) This work is licensed under a Creative Commons Attribution 4.0 International License. Read Full License 


\section{Abstract}

Background: Subclinical atherosclerosis develops in the thoracic aorta early in life. Insulin resistance is a well-established risk factor for the development of atherosclerosis. The triglyceride-glucose (TyG) index is a reliable marker of insulin resistance regardless of glucose tolerance or obesity. We aimed to investigate the relationship between the TyG index and aortic intima-media thickness (IMT) in patients without diabetes mellitus and cardiovascular disease undergoing transesophageal echocardiography due to suspected structural heart disease.

Methods: One hundred twenty-two patients enrolled in the study between January 2021 and June 2021. Triglyceride-glucose index was calculated using fasting laboratory parameters, and major cardiovascular risk factors and IMT of the thoracic aorta were recorded. Patients were divided into two groups according to their median IMT; patients with an IMT equal to or less than $1.42 \mathrm{~mm}$ were low IMT group, and higher than $1.42 \mathrm{~mm}$ were high IMT group.

Results: Triglyceride-glucose index were higher in the high IMT group ( $8.69 \pm 0.59$ vs. $8.37 \pm 0.53, p=$ 0.003). Triglyceride-glucose index was correlated with IMT $(r=0.259, p=0.005)$ and in binary logistic regression analysis age and TyG was independently associated with having high IMT $(\beta=0.076$, $\exp (B)=1.07995 \% C l=1.041-1.118, p<0.001$ and $\beta=0.847, \exp (B)=2.33495 \% C l=1.048-5.199, p=0.038$, respectively)

Conclusion: We found an independent relationship between TyG and IMT of the thoracic aorta, an early marker of subclinical atherosclerosis in patients without atherosclerotic cardiovascular disease. Therefore, the TyG index can identify high-risk subjects among healthy subjects.

\section{Introduction}

For a long time, it has been known that atherosclerosis begins early, including in utero, and manifests itself with some pathological processes before it becomes clinically evident [1]. Recognition of the development of atherosclerosis before it becomes a clinical disease is essential in identifying individuals at risk and deciding on whom to apply intensive treatment regimens [2,3]. However, the carotid artery is commonly used to measure preclinical atherosclerosis due to its availability; the thoracic aorta is affected by atherosclerosis earlier [4]. Patients who undergo transesophageal echocardiography for suspected structural heart disease such as atrial septal defect, ventricular septal defect, and thrombus in the left atrial appendage can be screened for markers of macroscopic disease and preclinical atherosclerosis of the thoracic aorta.

It is known that diabetes and metabolic syndrome, which are characterized by insulin resistance, accelerate the formation of all cardiovascular diseases, including coronary artery disease [2]. Numerous laboratory and anthropometric measurements have been proposed to measure insulin resistance $[5,6]$. Recently, the triglyceride-glucose (TyG) index has been used to measure insulin resistance. The triglyceride-glucose index is derived from blood levels of fasting glucose and triglycerides. It is calculated 
by taking the natural logarithm of half of the product of glucose and triglyceride levels. High availability and easy measurement make this index very useful in clinical practice. Regardless of glucose tolerance or obesity, the TyG index inversely correlated with insulin resistance and validated against the gold standard euglycemic-hyperinsulinemic clamp test [7]. As a good marker of insulin resistance, the TyG index is associated with an increased risk of diabetes mellitus [8], stroke [9], and coronary artery disease $[10,11]$ in healthy individuals. It is beneficial for estimating the risk of adverse prognosis of symptomatic and asymptomatic coronary artery disease and acute myocardial infarction [12-14]. In addition, there is evidence that the TyG index is also correlated with asymptomatic carotid artery atherosclerosis [15]. We aimed to examine the relationship between the TyG index and the intima-media thickness (IMT) of the thoracic aorta in patients who underwent transesophageal echocardiography for suspected structural heart disease such as atrioventricular septal defect, patent foramen ovale, and valvular heart diseases.

\section{Methods}

One hundred twenty-two patients who had undergone TEE between January 2021 and June 2021 for suspected structural heart disease such as atrial septal defect, ventricular septal defect, and thrombus in the left atrial appendage were enrolled in the study. Patients with hypertension, diabetes mellitus, coronary artery disease, peripheral artery disease, stroke, and chronic renal failure (eGFR $<30 \mathrm{ml} / \mathrm{min}$ ) were excluded from the study. Patients taking statins, fibrates, ezetimibe, PCSK9 inhibitors, antidiabetics, steroids, and other drugs that impact lipid characteristics or insulin sensitivity were also excluded from the study. Blood samples were drawn after 12 hours of fasting before the TEE procedure. Triglyceride-

glucose index was calculated using the formula; TyG $=\ln [($ Glucose $(\mathrm{mg} / \mathrm{dl})$ Triglycerides $(\mathrm{mg} / \mathrm{dl}) / 2]$. The estimated glomerular filtration rate was calculated using the new 2021 Chronic Kidney Disease Epidemiology Collaboration (CKD-EPI) creatinine equation [16]. Major risk factors for cardiovascular disease were recorded for each participant. The local ethics committee of the University of Health Sciences, Adana Training and Research Hospital approved the study design (meeting no: 87, issue no:1538). All participants carefully read and approved the informed consent. The study was conducted according to the Helsinki Declaration.

\section{Statistics}

SPSS 22.0 software (SPSS Inc. Chicago, IL) was used for statistical analysis. The Shapiro-Wilk test was used to analyze the normality of continuous variables. Continuous variables with a normal distribution were expressed as the mean, and standard deviation, while non-normally distributed variables were expressed as the median (minimum-maximum). Student's t-test and Mann-Whitney $U$ tests compared normally and non-normally distributed continuous variables between two groups. For bivariate analysis, Pearson and Spearman correlation coefficients were reported. Binary logistic regression analysis was used to determine the variables independently associated with IMT. The variables significantly associated with IMT were included in the regression model. We applied the receiver operating characteristics for testing the goodness of fit of our binary logistic regression analysis. A p-value less than 0.05 was considered statistically significant. 


\section{Transthoracic and Transesophageal Echocardiography}

Transthoracic echocardiography and TEE were performed using a commercially available system (Vivid 7R, GE Medical Systems, Horten, Norway). Following a 12-hour fasting interval, all patients underwent TEE with a $5 \mathrm{Mhz}$ multiplane transesophageal transducer. After oropharyngeal anesthesia with lidocaine spray, subjects were put in left decubitus with the left arm under their heads. The transducer was inserted through the mouth into the esophagus and gastric cavity to observe the cardiac and aortic structures. TEE was performed by an expert cardiologist who was blinded to other laboratory tests. All patients tolerated TEE well, and there were no complications. An expert observer independently interpreted all the recorded images. The thoracic aortic IMT was defined as the distance from the leading edge of the lumen-intima interface to the leading edge of the media-adventitia interface of the far wall. Intima-media thickness was measured manually in the thoracic aorta in six segments: ascending aorta, arcus aorta, and from distal arch to abdominal aorta was separated into four independent portions, each $5 \mathrm{~cm}$ long. The highest value for the IMT among the six segments was recorded [17].

\section{Results}

The study population is composed of 122 patients (mean age $40.39 \pm 15.29,65$ females $(53.27 \%)$ ). The study population was divided into two groups according to their median IMT. The first group was composed of 61 subjects (mean age $33.25 \pm 11.07$ years, 33 females $(50.8 \%)$ ) with IMT equal to or less than the median value of $1.42 \mathrm{~mm}$; the second group was composed of 61 subjects (mean age $47.52 \pm$ 15.67 years, 32 females (49.2\%)) greater than the median IMT. Atrial septal defect (39 (63.9\%) vs. 39 $(63.9 \%), p=1)$, atrial fibrillation (16 (26.2\%) vs. $15(24.6 \%), p=0.942)$ and valvular heart disease (6 (9.8\%) vs. $7(11.5 \%), p=0.894)$ were the indications of TEE (Table 1$)$.

Age, creatinine, glucose, triglyceride, and TyG index were higher in the high IMT group. Sex, smoking status, cholesterol levels, and TEE indications were similar among the groups (Table 1).

The IMT was correlated with age, creatinine, eGFR, glucose, triglycerides, creatinine, and TyG index. There was no correlation between cholesterol levels and IMT (Table 2). Age and eGFR were strongly correlated $(r=-0.841, p<0.001)$; therefore, eGFR was not included in the regression analysis with age to avoid multicollinearity.

Binary logistic regression analysis was performed to predict the variables associated with having high intima-media thickness. Creatinine, age, and TyG index were included in the regression model. In binary logistic regression analysis TyG index was positively related with IMT $(\beta=0.847, \exp (B)=2.33495 \% \mathrm{Cl}=$ $1.048-5.199, p=0.038)$. In addition, age was related with IMT $(\beta=0.076, \exp (B)=1.07995 \% \mathrm{Cl}=1.041$ $1.118, p<0.001$ ). Creatinine was not found to be significantly related to IMT (Table 3 ). Receiver operating characteristics curve analysis was applied for the binary logistic regression model for the equation logit $(p)=-10.316+0.076 *$ age $+0.847 *$ TyG index and revealed a high goodness of fit (AUC: $0.80895 \% \mathrm{Cl}$ : $0.728-0.888, p<0.001)$. 


\section{Discussion}

We showed that the TyG index is independently related to aortic IMT, a marker of subclinical atherosclerosis in healthy individuals without any previous cardiovascular disease or diabetes. Several investigations have linked the TyG index to ASCVD risk factors such as type 2 diabetes $[8,18]$, hypertension [19], and metabolic syndrome [20]. Other CVD surrogate measures related to the TyG index include coronary artery calcium score [21], IMT of carotid arteries, and arterial stiffness [22, 23]. A few studies have found a link between the TyG index and CVD in high-risk patients with diabetes and chronic renal disease [24-26]. To our knowledge, our study is the first to show an independent relationship between the TyG index and the thoracic aorta IMT. The Triglyceride-glucose index is directly proportional to the blood glucose and triglyceride levels, and both levels appear to be increased in individuals with insulin resistance. The causal relationship between insulin resistance and atherosclerosis is well established in the literature.

Examining the association between blood glucose and triglyceride levels separately with atherosclerosis can reveal the relationship between the TyG index and atherosclerosis. At the beginning of insulin resistance, increased lipolysis of stored triglycerides in adipose tissue and increased production of fatty acids in the liver increase triglyceride levels before elevating plasma glucose levels. Therefore, at the beginning of insulin resistance, fasting glucose remains in the normoglycemic range [27]. Several metaanalyses with many patients have shown that fasting plasma glucose levels, already within the normoglycemic range ( $<100 \mathrm{mg} / \mathrm{dL})$, are associated with the risk of developing coronary heart disease in subjects without diabetes mellitus. The risk of coronary disease increased linearly with blood glucose levels, independent of other established CVD risk factors, and the increased risk of diabetes [28] did not mediate it. Fasting glucose concentration is associated with coronary artery risk in people without diabetes, with an increased risk of $6 \%$ for every $1 \mathrm{mmol} / \mathrm{L}$ increment $(18 \mathrm{mg} / \mathrm{dL})$ in fasting plasma glucose, according to a meta-analysis of 26 prospective studies involving over 300,000 people [29].

Another finding in insulin resistance is elevated triglyceride levels, whose relationship with cardiovascular risk and mortality is disputed [30]. The difficulty in isolating hypertriglyceridemia's effect on cardiovascular disease is that it is frequently associated with other atherogenic lipid diseases, such as decreased HDL cholesterol and higher LDL cholesterol levels. In our study, we entered all lipid parameters in multivariate analysis to isolate the effect of triglycerides on IMT. However, in some studies, the relationship between triglyceride levels and cardiovascular disease became nonsignificant after adjusting for other lipid disorders and major variables; most studies found a direct association of triglyceride levels with increased risk and worse outcomes. Elevation of plasma free fatty acids activates the proinflammatory NFKB pathway [31], resulting in increased hepatic expression of several proinflammatory cytokines, including TNF-a, IL1- $\beta$, IL6, matrix metalloproteinases [32]; these inflammatory changes which play a significant role in the development of atherosclerosis [33]. It has been shown that daily administration of $2 \mathrm{~g}$ of icosapent ethyl reduces the frequency of cardiovascular death and ischemic events in patients who have recently used statins for coronary artery disease and still have high triglyceride levels $(135-499 \mathrm{mg} / \mathrm{dl})$. 
Since plaque thickness rather than morphology is thought to be a more objective and reliable indicator of atherosclerotic disease, most grading systems use the maximum plaque thickness of the most diseased segment. However, there are a few grading systems of aortic atheroma based on IMT; no standard or widely accepted IMT cut-off is available for classification [17]. We used the median value of $1.42 \mathrm{~mm}$ as a cut-off to indicate high IMT. In literature, IMT higher than $1.5 \mathrm{~mm}$ was associated with an increased risk of coronary artery disease and stroke [34]. Although our definition of having a high IMT may seem arbitrary, the median IMT value of the patients in our study is very close to the value known to increase the risk of coronary artery disease and stroke (1.42 vs. 1.5), provides a rational basis for our definition. Since atherosclerosis begins in the thoracic aorta earlier than in the carotid arteries $[4,35]$, our study has the advantage of delineating the relationship between $\mathrm{TyG}$ and subclinical atherosclerosis better than studies performed on the carotid arteries.

Lifestyle modifications or drug therapies are recommended for primary prevention by calculating the fatal and non-fatal cardiovascular disease risk using scoring systems (SCORE2 and SCORE2-OP) based on age, sex, blood pressure, smoking status, and non-HDL cholesterol levels [3]. In addition, some noninvasive tests like coronary artery calcium score $[36,37]$ and carotid ultrasound helps to reclassify individuals to a higher or lower risk category. Based on our findings, we may suggest that the TyG index can also be used for adding information on the calculated SCORE risk

Most of the subjects included in our study are young individuals (mean age $40.38 \pm 15.29$ years) with low cardiovascular risk. They represent a narrow area of the cardiovascular risk spectrum compared to the general population. In addition, the cross-sectional structure of our study makes it difficult to reveal the cause-effect relationship. These are major limitations of our study.

\section{Conclusion}

We showed that the TyG index predicts having a higher IMT independently of other major risk factors for atherosclerosis in healthy subjects without any cardiovascular disease. Based on this finding, the TyG index may be a useful marker to identify individuals at high risk of developing cardiovascular diseases.

\section{Abbreviations}

eGFR: Estimated glomerular filtration rate, IMT: Intima media thickness; TyG: Triglyceride glucose.

\section{Declarations}

\section{Acknowledgements}

None

\section{Authors' contributions}


SÖ created the idea, designed the study, did the statistical testing, wrote the manuscript text, and revised the text's language. GY contributed to the idea, criticized the study's design, and revised the manuscript. ÖDU collected the data and contributed to the statistical analysis.

\section{Funding}

None

\section{Availability of data and materials}

The datasets used and/or analyzed durıng the current study are available from the corresponding author upon reasonable request.

\section{Declarations}

\section{Ethics approval and consent to participate}

The local ethics committee of the University of Health Sciences, Adana Training and Research Hospital approved the study design (meeting no: 87 , issue no:1538). The study protocol was in accordance with the requirements of the Declarations of Helsinki of the World Medical Association. All participants gave written informed consent.

\section{Consent for Publication}

Not applicable.

\section{Competing interests}

The authors declare that they have no competing interests.

\section{References}

1. Palinski W, Napoli C. The fetal origins of atherosclerosis: maternal hypercholesterolemia, and cholesterol-lowering or antioxidant treatment during pregnancy influence in utero programming and postnatal susceptibility to atherogenesis. Faseb j. 2002,16:1348-60. https://doi.org/10.1096/fj.020226rev.

2. Beverly JK, Budoff MJ. Atherosclerosis: Pathophysiology of insulin resistance, hyperglycemia, hyperlipidemia, and inflammation. J Diabetes. 2020, 12:102-04. https://doi.org/10.1111/17530407.12970.

3. Visseren FLJ, Mach F, Smulders YM, Carballo D, Koskinas KC, Back M, Benetos A, Biffi A, Boavida JM, Capodanno D, et al: 2021 ESC Guidelines on cardiovascular disease prevention in clinical practice. Eur Heart J 2021, 42:3227-337. https://doi.org/10.1093/eurheartj/ehab484. 
4. Harrington J, Peña AS, Gent R, Hirte C, Couper J. Aortic intima-media thickness is an early marker of atherosclerosis in children with type 1 diabetes mellitus. J Pediatr. 2010, 156:237-41. https://doi.org/10.1016/j.jpeds.2009.08.036.

5. Khan SH, Sobia F, Niazi NK, Manzoor SM, Fazal N, Ahmad F. Metabolic clustering of risk factors: evaluation of Triglyceride-glucose index (TyG index) for evaluation of insulin resistance. Diabetology \& Metabolic Syndrome. 2018, 10. https://doi.org/10.1186/s13098-018-0376-8.

6. Du T, Yuan G, Zhang M, Zhou X, Sun X, Yu X. Clinical usefulness of lipid ratios, visceral adiposity indicators, and the triglycerides and glucose index as risk markers of insulin resistance.

Cardiovascular Diabetology. 2014, 13. https://doi.org/10.1186/s12933-014-0146-3.

7. Guerrero-Romero F, Simental-Mendía LE, González-Ortiz M, Martínez-Abundis E, Ramos-Zavala MG, Hernández-González SO, Jacques-Camarena $\mathrm{O}$, Rodríguez-Morán M: The product of triglycerides and glucose, a simple measure of insulin sensitivity. Comparison with the euglycemic-hyperinsulinemic clamp. J Clin Endocrinol Metab. 2010, 95:3347-51. https://doi.org/10.1210/jc.2010-0288.

8. da Silva A, Caldas APS, Rocha D, Bressan J. Triglyceride-glucose index predicts independently type 2 diabetes mellitus risk: A systematic review and meta-analysis of cohort studies. Prim Care Diabetes. 2020, 14:584-93. https://doi.org/10.1016/j.pcd.2020.09.001.

9. Shi W, Xing L, Jing L, Tian Y, Yan H, Sun Q, Dai D, Shi L, Liu S. Value of triglyceride-glucose index for the estimation of ischemic stroke risk: Insights from a general population. Nutr Metab Cardiovasc Dis. 2020, 30:245-53. https://doi.org/10.1016/j.numecd.2019.09.015.

10. Da Silva A, Caldas APS, Hermsdorff HHM, Bersch-Ferreira ÂC, Torreglosa CR, Weber B, Bressan J. Triglyceride-glucose index is associated with symptomatic coronary artery disease in patients in secondary care. Cardiovascular Diabetology. 2019, 18. https://doi.org/10.1186/s12933-019-0893-2.

11. Park GM, Cho YR, Won KB, Yang YJ, Park S, Ann SH, Kim YG, Park EJ, Kim SJ, Lee SG, et al: Triglyceride glucose index is a useful marker for predicting subclinical coronary artery disease in the absence of traditional risk factors. Lipids Health Dis. 2020, 19:7. https:// doi.org/10.1186/s12944020-1187-0.

12. Tian X, Zuo Y, Chen S, Liu Q, Tao B, Wu S, Wang A. Triglyceride-glucose index is associated with the risk of myocardial infarction: an 11-year prospective study in the Kailuan cohort. Cardiovasc Diabetol. 2021, 20:19. https://doi.org/10.1186/s12933-020-01210-5.

13. Zhang Y, Ding X, Hua B, Liu Q, Gao H, Chen H, Zhao X-Q, Li W, Li H. High triglyceride-glucose index is associated with adverse cardiovascular outcomes in patients with acute myocardial infarction. Nutrition, Metabolism and Cardiovascular Diseases. 2020,30:2351-62. https://doi.org/10.1016/j.numecd.2020.07.041.

14. Meireles DP, Santos IS, Alencar AP, Lotufo PA, Benseñor IM, Goulart AC. Prognostic value of carotid intima-media in the short- and long-term mortality in the Strategy of Registry of Acute Coronary Syndrome (ERICO) study. Echocardiography. 2018,35:1351-61. https://doi.org/10.1111/echo.14044.

15. Irace C, Carallo C, Scavelli FB, De Franceschi MS, Esposito T, Tripolino C, Gnasso A. Markers of insulin resistance and carotid atherosclerosis. A comparison of the homeostasis model assessment 
and triglyceride glucose index. Int J Clin Pract. 2013, 67:665-72. https://doi.org/10.1111/ijcp.12124.

16. Inker LA, Eneanya ND, Coresh J, Tighiouart H, Wang D, Sang Y, Crews DC, Doria A, Estrella MM, Froissart M, et al. New Creatinine- and Cystatin C-Based Equations to Estimate GFR without Race. N Engl J Med 2021, 385:1737-49. https://doi.org/10.1056/NEJMoa2102953.

17. Goldstein SA, Evangelista A, Abbara S, Arai A, Asch FM, Badano LP, Bolen MA, Connolly HM, CuellarCalabria $\mathrm{H}$, Czerny $\mathrm{M}$, et al: Multimodality imaging of diseases of the thoracic aorta in adults: from the American Society of Echocardiography and the European Association of Cardiovascular Imaging: endorsed by the Society of Cardiovascular Computed Tomography and Society for Cardiovascular Magnetic Resonance. J Am Soc Echocardiogr. 2015, 28:119-82. https://doi.org/10.1016/j.echo.2014.11.015.

18. Wang X, Liu J, Cheng Z, Zhong Y, Chen X, Song W. Triglyceride glucose-body mass index and the risk of diabetes: a general population-based cohort study. Lipids Health Dis. 2021, 20:99. https://doi.org/10.1186/s12944-021-01532-7.

19. Jian S, Su-Mei N, Xue C, Jie Z, Xue-Sen W. Association and interaction between triglyceride-glucose index and obesity on risk of hypertension in middle-aged and elderly adults. Clin Exp Hypertens. 2017, 39:732-39. https://doi.org/10.1080/10641963.2017.1324477.

20. Angoorani P, Heshmat R, Ejtahed HS, Motlagh ME, Ziaodini H, Taheri M, Aminaee T, Goodarzi A, Qorbani M, Kelishadi R. Validity of triglyceride-glucose index as an indicator for metabolic syndrome in children and adolescents: the CASPIAN-V study. Eat Weight Disord. 2018, 23:877-83. https://doi.org/10.1007/s40519-018-0488-z.

21. Kim MK, Ahn CW, Kang S, Nam JS, Kim KR, Park JS. Relationship between the triglyceride glucose index and coronary artery calcification in Korean adults. Cardiovascular Diabetology. 2017, 16. https://doi.org/10.1186/s12933-017-0589-4.

22. Won K-B, Park G-M, Lee S-E, Cho I-J, Kim HC, Lee BK, Chang H-J. Relationship of insulin resistance estimated by triglyceride glucose index to arterial stiffness. Lipids in Health and Disease. 2018, 17. https://doi.org/10.1186/s12944-018-0914-2.

23. Baydar O, Kilic A, Okcuoglu J, Apaydin Z, Can MM. The Triglyceride-Glucose Index, a Predictor of Insulin Resistance, Is Associated With Subclinical Atherosclerosis. Angiology, 2021, 72: 994-1000. https://doi.org/10.1177/00033197211007719.

24. Jin JL, Sun D, Cao YX, Guo YL, Wu NQ, Zhu CG, Gao Y, Dong QT, Zhang HW, Liu G, et al. Triglyceride glucose and haemoglobin glycation index for predicting outcomes in diabetes patients with newonset, stable coronary artery disease: a nested case-control study. Ann Med. 2018, 50:576-86. https://doi.org/10.1080/07853890.2018.1523549.

25. Yan Z, Yu D, Cai Y, Shang J, Qin R, Xiao J, Zhao B, Zhao Z, Simmons D. Triglyceride Glucose Index Predicting Cardiovascular Mortality in Chinese Initiating Peritoneal Dialysis: A Cohort Study. Kidney and Blood Pressure Research. 2019, 44:669-78. https://doi.org/10.1159/000500979.

26. Thai PV, Tien HA, Van Minh H, Valensi P. Triglyceride glucose index for the detection of asymptomatic coronary artery stenosis in patients with type 2 diabetes. Cardiovascular Diabetology. 
2020, 19:137. https://doi.org/10.1186/s12933-020-01108-2.

27. Tenenbaum A, Klempfner R, Fisman EZ. Hypertriglyceridemia: a too long unfairly neglected major cardiovascular risk factor. Cardiovasc Diabetol. 2014, 13:159. https://doi.org/10.1186/s12933-0140159-y.

28. Shaye K, Amir T, Shlomo S, Yechezkel S. Fasting glucose levels within the high normal range predict cardiovascular outcome. Am Heart J. 2012, 164:111-6. https://doi.org/10.1016/j.ahj.2012.03.023.

29. Sarwar N, Aspelund T, Eiriksdottir G, Gobin R, Seshasai SR, Forouhi NG, Sigurdsson G, Danesh J, Gudnason V. Markers of dysglycaemia and risk of coronary heart disease in people without diabetes: Reykjavik prospective study and systematic review. PLoS Med. 2010, 7:e1000278. https://doi.org/10.1371/journal.pmed.1000278.

30. Petersen MC, Shulman GI. Mechanisms of Insulin Action and Insulin Resistance. Physiol Rev. 2018, 98:2133-223. https://doi.org/10.1152/physrev.00063.2017.

31. Barazzoni R, Zanetti M, Cappellari GG, Semolic A, Boschelle M, Codarin E, Pirulli A, Cattin L, Guarnieri G. Fatty acids acutely enhance insulin-induced oxidative stress and cause insulin resistance by increasing mitochondrial reactive oxygen species (ROS) generation and nuclear factor-kB inhibitor (IKB) -nuclear factor-KB (NFKB) activation in rat muscle, in the. Diabetologia. 2012, 55:773-82. https://doi.org/10.1007/s00125-011-2396-x.

32. Longo GM, Xiong W, Greiner TC, Zhao Y, Fiotti N, Baxter BT. Matrix metalloproteinases 2 and 9 work in concert to produce aortic aneurysms. J Clin Invest. 2002, 110:625-32. https://doi.org/10.1172/jci15334.

33. Xu H, Barnes GT, Yang Q, Tan G, Yang D, Chou CJ, Sole J, Nichols A, Ross JS, Tartaglia LA, Chen H. Chronic inflammation in fat plays a crucial role in the development of obesity-related insulin resistance. Journal of Clinical Investigation. 2003, 112:1821-30. https://doi.org/10.1172/jci19451.

34. Stein JH, Korcarz CE, Hurst RT, Lonn E, Kendall CB, Mohler ER, Najjar SS, Rembold CM, Post WS. Use of carotid ultrasound to identify subclinical vascular disease and evaluate cardiovascular disease risk: a consensus statement from the American Society of Echocardiography Carotid Intima-Media Thickness Task Force. Endorsed by the Society for Vascular Medicine. J Am Soc Echocardiogr. 2008, 21:93-111; quiz 89-90. https://doi.org/10.1016/j.echo.2007.11.011.

35. McGill HC, Jr., McMahan CA, Herderick EE, Tracy RE, Malcom GT, Zieske AW, Strong JP. Effects of coronary heart disease risk factors on atherosclerosis of selected regions of the aorta and right coronary artery. PDAY Research Group. Pathobiological Determinants of Atherosclerosis in Youth. Arterioscler Thromb Vasc Biol. 2000, 20:836-45. https://doi.org/10.1161/01.atv.20.3.836.

36. Lin JS, Evans CV, Johnson E, Redmond N, Coppola EL, Smith N. Nontraditional Risk Factors in Cardiovascular Disease Risk Assessment: Updated Evidence Report and Systematic Review for the US Preventive Services Task Force. Jama. 2018, 320:281-97. https://doi.org/10.1001/jama.2018.4242.

37. Peters SA, den Ruijter HM, Bots ML, Moons KG. Improvements in risk stratification for the occurrence of cardiovascular disease by imaging subclinical atherosclerosis: a systematic review. Heart. 2012, 
98:177-84. https://doi.org/10.1136/heartjnl-2011-300747.

\title{
Tables
}

\author{
Table 1
}

Comparison of IMT groups 
IMT low group $n=61 \quad$ IMT high group $n=61 \quad p$

\begin{tabular}{|c|c|c|c|c|c|c|c|}
\hline Age & 33.25 & \pm & 11.07 & 47.52 & \pm & 15.67 & $<0.001$ \\
\hline Sex (female), n (\%) & \multicolumn{3}{|c|}{$33(50.8 \%)$} & \multicolumn{3}{|c|}{$32(49.2)$} & 1 \\
\hline Smoking, n (\%) & \multicolumn{3}{|c|}{$14(23 \%)$} & \multicolumn{3}{|c|}{$13(21.3)$} & 1 \\
\hline Systolic BP, mmHg & 122.42 & \pm & 11.44 & 123.56 & \pm & 10.98 & 0.572 \\
\hline Diastolic BP, mmHg & 81.34 & \pm & 8.67 & 82.42 & \pm & 7.92 & 0.474 \\
\hline Waist to hip ratio & 0.92 & \pm & 0.09 & 0.9 & \pm & 0.09 & 0.22 \\
\hline Body mass index, $\mathrm{kg} / \mathrm{m}^{2}$ & 26.1 & \pm & 3.12 & 25.88 & \pm & 3.48 & 0.713 \\
\hline Ejection fraction, \% & 62.65 & \pm & 3.51 & 62.62 & \pm & 3.98 & 0.969 \\
\hline Hemoglobin, (gr/dl) & 13.88 & \pm & 2.88 & 13.8 & \pm & 1.82 & 0.077 \\
\hline BUN & 24.1 & \pm & 7.03 & 27.64 & \pm & 10.73 & 0.068 \\
\hline Creatinine, (mg/dl) & 0.74 & \pm & 0.15 & 0.8 & \pm & 0.2 & 0.042 \\
\hline eGFR $\left(\mathrm{ml} / \mathrm{min} / 1.73 \mathrm{~m}^{2}\right)$ & 105 & \pm & 12.52 & 90.42 & \pm & 18.24 & $<0.001$ \\
\hline Glucose (mg/dl) & 87.73 & \pm & 11.1 & 98.45 & \pm & 25.41 & 0.004 \\
\hline Total Cholesterol, (mg/dl) & 175 & \pm & 38.97 & 185.36 & \pm & 42.4 & 0.169 \\
\hline LDL-Cholesterol, (mg/dl) & 109.02 & \pm & 33.62 & 117.84 & \pm & 34.21 & 0.159 \\
\hline HDL- Cholesterol, (mg/dl) & 46.6 & \pm & 13.17 & 43.37 & \pm & 10.34 & 0.141 \\
\hline Triglycerides, (mg/dl) & \multicolumn{3}{|c|}{$91(28-329)$} & \multicolumn{3}{|c|}{$113(29.3-397)$} & 0.006 \\
\hline Triglyceride-Glucose Index & 8.37 & \pm & 0.53 & 8.69 & \pm & 0.59 & 0.003 \\
\hline \multicolumn{8}{|l|}{ Indication of TEE } \\
\hline Atrial septal defect, n, \% & \multicolumn{3}{|c|}{$39(63.9 \%)$} & \multicolumn{3}{|c|}{$39(63.9 \%)$} & 0.947 \\
\hline Atrial fibrillation, n, \% & \multicolumn{3}{|c|}{$16(26.2 \%)$} & \multicolumn{3}{|c|}{$15(24.6 \%)$} & 0.947 \\
\hline Valvular disease, $n, \%$ & \multicolumn{3}{|c|}{$6(9.8 \%)$} & \multicolumn{3}{|c|}{$7(11.5 \%)$} & 0.894 \\
\hline
\end{tabular}

The variables were expressed as mean \pm standard deviation and median (minimum-maximum), BP: Blood pressure, eGFR: Estimated glomerular filtration rate, IMT: Intima-media thickness, TEE:

Transesophageal echocardiography. Students t-test and Kruskal Wallis tests were used 


\begin{tabular}{lll} 
& $\mathrm{r}$ & $\mathrm{p}$ \\
\hline Age, years & 0.564 & $<0.001$ \\
\hline Creatinine $(\mathrm{mg} / \mathrm{dl})$ & 0.230 & 0.012 \\
\hline eGFR $\left(\mathrm{mil} / \mathrm{min} / \mathrm{m}^{2}\right)$ & -0.462 & $<0.001$ \\
\hline Total Cholesterol, $(\mathrm{mg} / \mathrm{dl})$ & 0.116 & 0.213 \\
\hline LDL Cholesterol, $(\mathrm{mg} / \mathrm{dl})$ & 0.146 & 0.115 \\
\hline HDL Cholesterol, $(\mathrm{mg} / \mathrm{dl})$ & -0.078 & 0.402 \\
\hline Triglyceride Glucose $\mathrm{Index}$ & 0.259 & 0.005
\end{tabular}

eGFR: Estimated glomerular filtration rate. Pearson and Spearman correlation tests were used.

Table 3

Binary logistic regression analysis for having high Intima-media thickness.

\begin{tabular}{|lllll|}
\hline & $\mathrm{B}$ & $\operatorname{Exp}(\mathrm{B})$ & $95 \% \mathrm{Cl}$ for $\operatorname{EXP}(\mathrm{B})$ & $\mathrm{p}$ \\
\hline Age, years & 0.076 & 1.079 & $1.041-1.118$ & $<0.001$ \\
\hline Creatinine, $(\mathrm{mg} / \mathrm{dl})$ & 0.012 & 1.012 & $0.081-12.619$ & 0.992 \\
\hline Triglyceride Glucose Index & 0.847 & 2.334 & $1.048-5.199$ & 0.038 \\
\hline Constant & -10.316 & & & \\
\hline
\end{tabular}

Nagelkerke $\mathrm{R}^{2}=0.350$. Binary logistic regression analysis was used. 